\title{
The explosion sites of nearby supernovae seen with integral field spectroscopy
}

\author{
Hanindyo Kuncarayakti ${ }^{1,2}$ \\ ${ }^{1}$ Millennium Institute of Astrophysics (MAS), Santiago, Chile \\ ${ }^{2}$ Departamento de Astronomía, Universidad de Chile \\ Casilla 36-D, Santiago, Chile \\ email: hanin@das.uchile.cl
}

\begin{abstract}
Integral field spectroscopy of nearby supernova sites within $\sim 30 \mathrm{Mpc}$ have been obtained using multiple IFU spectrographs in Hawaii and Chile. This technique enables both spatial and spectral information of the explosion sites to be acquired simultaneously, thus providing the identification of the parent stellar population of the supernova progenitor and the estimates for its physical parameters including age and metallicity via the spectrum. While this work has mainly been done in the optical wavelengths using instruments such as VIMOS, GMOS, and MUSE, a near-infrared approach has also been carried out using the AO-assisted SINFONI. By studying the supernova parent stellar population, we aim to characterize the mass and metallicity of the progenitors of different types of supernovae.
\end{abstract}

Keywords. Keyword1, keyword2, keyword3, etc.

Despite the increasingly high number of supernova (SN) discoveries, there is still limited understanding on their progenitor stars. Both direct and indirect studies contribute to our understanding by providing important clues about the nature of these objects. A number of direct detections of the progenitor stars have been achieved by sifting archival high-resolution images, mostly from the Hubble Space Telescope (see Elias-Rosa 2015). Alternatively, one may perform statistical studies on the supernova environments to derive useful constraints on the SN progenitor star (Anderson et al. 2015).

Extending the study published earlier in Kuncarayakti et al. (2013a,b), we conducted integral field spectroscopy of nearby $(\lesssim 30 \mathrm{Mpc})$ core-collapse SN sites observable from the southern hemisphere. Using VLT, Gemini, and Magellan telescopes equipped with IFU spectrographs, the observations have been done and the initial sample presented in Kuncarayakti et al. (2013a,b) has now been expanded to four times its original size.

We extracted the spectra of the parent stellar populations of the SN progenitors at each explosion sites, and used Starburst99 SSP models (Leitherer et al. 1999) to infer the age via $\mathrm{H} \alpha$ emission equivalent width. Metallicity was measured using strong-line method (Pettini \& Pagel 2004). Our preliminary result derived from a part of the sample indicates that there is no statistically significant relation observed between the SN types (II/Ib/Ic) and the age/metallicity of the parent stellar population. Taking the age as the progenitor star lifespan, the initial mass of the star was estimated and in Fig. 1 plotted on the mass-metallicity diagram. Similar to Kuncarayakti et al. (2013a,b), there is no preference for a SN type to occupy a particular place on the mass-metallicity plane.

Employing AO-assisted infrared IFU observations, we further resolve a subset of the explosion sites to study the star formation history at the explosion sites. Comparing different age indicators, we do not see correlating spatial distributions. This may be interpreted as an effectively instantaneous star formation. With the complete data sample we envision a full analysis to be published soon (Kuncarayakti et al., in preparation). 


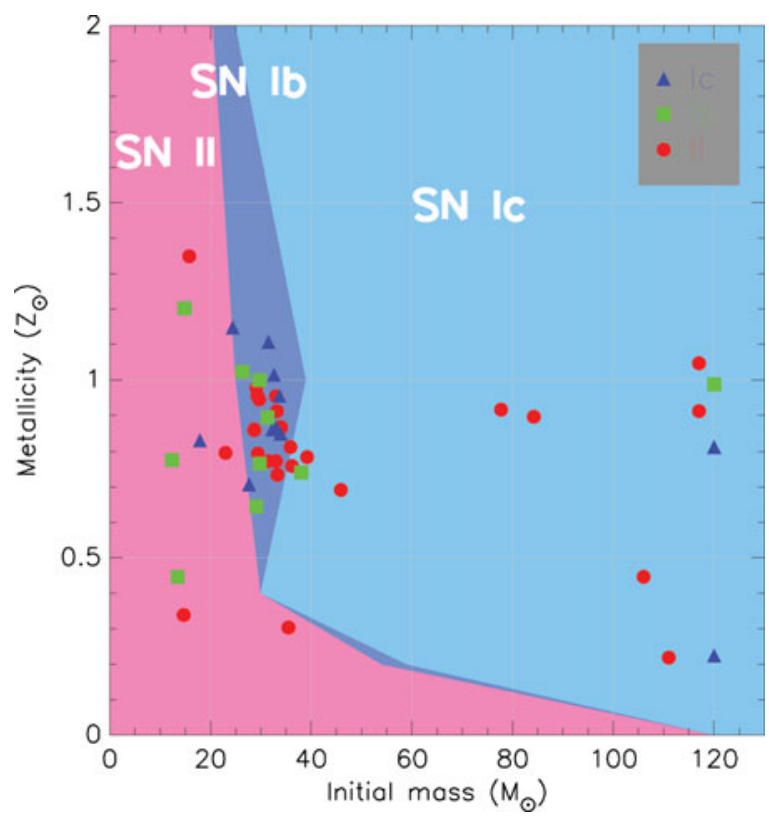

Figure 1. Updated mass-metallicity diagram of Kuncarayakti et al. (2013a,b), showing SN progenitors compared to the single-star progenitor models (Georgy et al. 2009, shaded areas).

\section{Acknowledgement}

Support for HK is provided by the Ministry of Economy, Development, and Tourism's Millennium Science Initiative through grant IC120009, awarded to The Millennium Institute of Astrophysics, MAS. HK acknowledges support by CONICYT through FONDECYT grant 3140563.

\section{References}

Anderson, J. P., James, P. A., Habergham, S. M., Galbany, L., \& Kuncarayakti, H. 2015, PASA, $32, \mathrm{e} 019$

Elias-Rosa, N. 2015, this volume

Georgy, C., Meynet, G., Walder, R., Folini, D., \& Maeder, A. 2009, AधチA, 502, 611

Kuncarayakti, H., Doi, M., Aldering, G., et al. 2013, AJ, 146, 30

Kuncarayakti, H., Doi, M., Aldering, G., et al. 2013, AJ, 146, 31

Leitherer, C., Schaerer, D., Goldader, J. D., et al. 1999, ApJS, 123, 3

Pettini, M. \& Pagel, B. E. J. 2004, MNRAS, 348, L59 